\title{
Chikungunya Virus and the Mosquito Vector Aedes aegypti in New Caledonia (South Pacific Region)
}

\author{
Myrielle Dupont-Rouzeyrol, ${ }^{1}$ Valérie Caro, ${ }^{2}$ Laurent Guillaumot, ${ }^{3}$ Marie Vazeille, ${ }^{4}$ \\ Eric D'Ortenzio, ${ }^{5}$ Jean-Michel Thiberge, ${ }^{2}$ Noémie Baroux, ${ }^{5}$ Ann-Claire Gourinat, ${ }^{6}$ \\ Marc Grandadam, ${ }^{7,8, *}$ and Anna-Bella Failloux ${ }^{4, *}$
}

\begin{abstract}
Chikungunya virus (CHIKV) is transmitted to humans through the bite of Aedes mosquitoes. During the 20052006 epidemic that occurred in the Indian Ocean Islands, a viral strain harboring a substitution of an alanine to valine at position 226 (E1-A226V) of the E1 glycoprotein enhanced the transmissibility of CHIKV by Aedes albopictus. In March 2011, autochthonous transmission of CHIKV was reported in New Caledonia (NC), an island located in the southwest Pacific Ocean. This was the first report of local chikungunya (CHIK) transmission in this region of the world. Phylogenetic analysis based on the complete genome demonstrated that the CHIKV-NC strain isolated from the first autochthonous human case belongs to the Asian lineage. This is consistent with the Indonesian origin of CHIK cases previously imported and detected. Thus the CHIKV-NC does not present a valine substitution at position E1-226. In New Caledonia, the putative vector of CHIKV is Aedes aegypti, since no other potential vector has ever been described. For example, A. albopictus is not found in NC. Vector competence experiments showed that $A$. aegypti from New Caledonia was able to transmit, as early as 3 days post-infection, two CHIKV strains: CHIKV-NC belonging to the Asian lineage, and CHIKV-RE from Reunion Island harboring the E1-A226V mutation. Thus the extrinsic incubation period of both CHIKV strains in this vector species could be considered to be quite short. These results illustrate the threat of the spread of CHIKV in the South Pacific region. From February to June 2011 (the end of the alert), only 33 cases were detected. Implementation of drastic vector control measures and the occurrence of the cold season probably helped to limit the extent of the outbreak, but other factors may have also been involved and are discussed.
\end{abstract}

Key Words: Aedes aegypti-Arboviruses—Chikungunya-New Caledonia-Vector competence.

\section{Introduction}

C hikungunya fever is an acute illness caused by chikungunya virus (CHIKV), an alphavirus of the family Togaviridae. CHIKV is transmitted to humans through the bite of infected Aedes species mosquitoes, particularly Aedes aegypti and Aedes albopictus. It induces an arthro-febrile syndrome affecting mostly the extremities, and is associated with polyarthritis and skin rash (reviewed in Schwartz and Albert 2010). The clinical illness is often associated with prolonged morbidity, and significant economic and social consequences (Enserink 2007). Outbreaks of chikungunya (CHIK) have been

\footnotetext{
${ }^{1}$ Institut Pasteur de Nouvelle-Calédonie, Réseau International des Instituts Pasteur, Laboratoire d'Epidémiologie Moléculaire, Nouméa, New Caledonia.

${ }^{2}$ Institut Pasteur, Plate-forme Génotypage des Pathogènes et Santé Publique, Paris, France.

${ }^{3}$ Institut Pasteur de Nouvelle-Calédonie, Réseau International des Instituts Pasteur, Laboratoire d'Entomologie Médicale, Nouméa, New Caledonia.

${ }^{4}$ Institut Pasteur, Unité de Génétique Moléculaire des Bunyavirus, Paris, France.

${ }^{5}$ Institut Pasteur de Nouvelle-Calédonie, Réseau International des Instituts Pasteur, Unité d’Epidémiologie des Maladies Infectieuses, Nouméa, New Caledonia.

${ }^{6}$ Institut Pasteur de Nouvelle-Calédonie, Réseau International des Instituts Pasteur, Laboratoire de Diagnostic Spécialisé, Nouméa, New Caledonia.

${ }^{7}$ Institut Pasteur, CNR des Arbovirus, Paris, France.

${ }^{8}$ Institut Pasteur of Lao PDR, Institut Pasteur International Network, Ventiane, Lao PDR.

*These authors contributed equally to this study.
} 
described mainly in Africa and Asia, but the disease has recently emerged in other parts of the world after carriage by infected travelers, and CHIK is becoming a worldwide public health problem. Phylogenetic analyses have demonstrated three distinct lineages of CHIKV strains: West Africa, Asia, and East/Central/South Africa (ECSA). In 2005 and 2006, the Indian Ocean region was hit by an extraordinarily large CHIK epidemic that reached India and Southeast Asia (Hapuarachchi et al. 2010; Volk et al. 2010). The circulating CHIKV strains from the Indian Ocean islands are derived from the ECSA group. Most strains harbor a substitution from an alanine to a valine at position 226 of the CHIKV E1 glycoprotein. This substitution has been demonstrated to favor CHIKV transmission by Ae. albopictus, but not by Ae. aegypti (Schuffenecker et al. 2006; Vazeille et al. 2007; Tsetsarkin et al. 2007; de Lamballerie et al. 2008). Since the 2005-2006 Indian Ocean outbreak, CHIKV strains from the ECSA group harboring the substitution E1-A226V and Ae. albopictus have been extensively implicated (reviewed in Schwartz and Albert 2010). Indeed in 2007 in Europe, the first outbreak of CHIK occurred in northeastern Italy, and resulted in 205 human cases. The CHIKV strain was assigned to the ECSA group harboring the substitution E1-A226V. The vector incriminated was Ae. albopictus (Rezza et al. 2007). Thus unexpectedly, in September 2010, autochthonous cases of CHIK were detected in southeastern France due to viral strains belonging to the ECSA group, which presented an alanine in E1-226 (Grandadam et al. 2011). Ae. albopictus was the likely vector. These two European CHIK episodes underscore the dissemination potential of CHIK in Europe and in other countries where Ae. albopictus is present (reviewed in Paupy et al. 2009).

Local CHIKV transmission has never been reported in the South Pacific region. The first outbreak occurred in New Caledonia (NC) from February to June 2011 (Alibert et al. 2011). In this study, we identified the genotype of a CHIKV strain isolated from the first autochthonous case in NC, and assessed its transmission efficiency by Ae. aegypti populations from NC.

\section{Materials and Methods}

\section{Study area}

New Caledonia is an island located in the Western Pacific region between latitude $20^{\circ}$ and $22^{\circ}$ south, approximately $2000 \mathrm{~km}$ northeast of Sydney, Australia. The main city is Noumea, located in the south on the western coast. About half of the 250,000 New Caledonians live in Noumea and its surrounding area. NC has a tropical climate with two marked seasons: December to March being warm and wet (monthly mean temperature between 26 and $27^{\circ} \mathrm{C}$ ), and June to September being dry and much cooler (monthly mean temperature between 19 and $21^{\circ} \mathrm{C}$ ).

\section{Laboratory diagnosis and case definitions}

Serum samples collected after 5 days following the onset of illness were tested for IgM antibody to CHIKV with IgM immunocapture assays (MAC-ELISA; Pastorino et al. 2004). Antigens for those tests were prepared by the French National Reference Centre for Arboviruses, Institut Pasteur, Paris, France (CNR des Arbovirus). For IgM-positive cases, convalescent sera was collected 2 weeks after the first sampling and analyzed for CHIKV-specific IgG antibodies by an ELISA de- veloped at the CNR des Arbovirus. RT-PCR detection of the CHIKV genome was performed on blood during the first 6 days of infection, using the Superscript One-Step RT-PCR with platinum Taq kit (Invitrogen, Carlsbad, CA; Pastorino et al. 2005), on LC480 (Roche Diagnostics, Mannheim, Germany), after RNA extraction with the EasyMag system (bioMérieux, Paris, France). Considering the possibility of clinical confusion with other viruses belonging to the Semliki Forest complex present in the area, the first early sera were also tested for Ross River virus (RRV) by real-time RT-PCR (Hall et al. 2010).

Serum samples were also tested for dengue virus infection using IgM antibody capture ELISA (PanBio, Brisbane, Australia), and NS1 Platelia antigen detection (BioRad, Marnesla-Coquette, France).

A clinically supported case was defined as a patient presenting with sustained fever associated with arthralgia or arthritis. A laboratory-positive case was defined as the presence of viral RNA by RT-PCR, or a seroconversion or a significant rise (fourfold) in antibody level. A probable case was defined as a patient positive for virus-specific IgM antibodies in a single serum sample collected at the acute or convalescent stage.

\section{Origin of the New Caledonian CHIKV strain studied}

The first two cases were serologically diagnosed and related to individuals returning from Indonesia at the beginning of February 2011. The first case (patient A), diagnosed on February 25, 2011, lives in rural NC and did not cause any secondary cases. The second case (patient B), identified after interviewing patient A, lives in Noumea (Alibert et al. 2011). Patient C, diagnosed on March 3, 2011 using RT-PCR (RRV RT-PCR negative and dengue NS1 negative), lives near patient B, and was the first autochthonous CHIK case from whom the CHIKV was cultured and analyzed. By late June 2011, 33 confirmed cases had been investigated by the health authorities (Alibert et al. 2011). Virus transmission was apparently interrupted after this date.

\section{Phylogenetic analyses}

Viral genomic RNA was extracted from CHIKV grown once on mosquito C6/36 cells. RT-PCR was performed using SuperScript One-Step RT-PCR with platinum Taq (Invitrogen), with primers targeting the complete genome (Schuffenecker et al. 2006). RT-PCR fragments were purified by ultrafiltration. Sequencing reactions were performed using the Big Dye Terminator v1.1 cycle sequencing kit (Applied Biosystems, Foster City, $\mathrm{CA}$ ). Sequence chromatograms from both strands were obtained on automated sequence analyzer ABI3730XL (Applied Biosystems). For sequence analysis, contig assembly and sequence alignments were performed using program BioNumerics version 6.5 (Applied-Maths, Sint-Martens-Latem, Belgium). For phylogenetic analysis, a maximum-likelihood tree was constructed using MEGA version 5 software (www.megasoftware .net), based on the Tamura-Nei model. The reliability of nodes was assessed by bootstrap resampling with 1000 replicates.

\section{Vector control during the outbreak and entomological investigation}

The day after the report of the first cases, intensive vector control measures were implemented. These measures included thorough and repeated source reduction carried out 
by city council staff, in addition to adulticide sprayings in the dwellings of confirmed cases and within a 200-m radius. Despite these measures, other cases were confirmed in the residential district of patients $B$ and $C$, and later the disease spread to other areas of Noumea and the neighboring township of Dumbea, where similar vector control measures were also implemented (Alibert et al. 2011).

Ae. aegypti populations were collected at immature stages in March 2011 in two areas of Noumea, one close to the first transmission cluster, and the second in another district. Mosquitoes were reared in a laboratory to obtain eggs for further experiments. As a routine measure carried out in the context of vector surveillance, susceptibility of the two mosquito populations to deltamethrin was tested following the World Health Organization (WHO) standard impregnated paper protocol (World Health Organization 1981). A resistant strain had previously been established in the laboratory from a sample collected in 2010, and was subsequently selected for resistance by exposing individuals three times to the insecticide. The deltamethrin-resistant strain was included in the study to detect any possible correlation between vector competence and insecticide resistance, as suggested by Rivero and associates (2010).

\section{Vector competence experiments}

First-generation (F1) individuals were used for vector competence experiments using two CHIKV strains displaying different genetic patterns and different potentials of transmission by Ae. albopictus (Tsetsarkin et al. 2007; Vazeille et al. 2007): CHIKV-NC, presenting an alanine residue at E1-226, and
CHIKV-RE from Reunion Island (2005), harboring the mutation A226V in the E1 glycoprotein (named 06.21 in Schuffenecker et al. 2006). F1 females were allowed to feed on an infectious blood meal at a titer of $10^{7} \mathrm{pfu} / \mathrm{mL}$ provided in a glass feeder maintained at $37^{\circ} \mathrm{C}$. The infectious meal was composed of a virus suspension diluted (1:3) in washed rabbit erythrocytes (Vazeille-Falcoz et al. 1999). The titer used was close to the patients' viremic level (Parola et al. 2006). Engorged females were sorted on ice and incubated at $28^{\circ} \mathrm{C}$ as described in Vazeille and associates (2007). This temperature is close to the monthly average warm season temperature (i.e., January $26.9^{\circ} \mathrm{C}$, February $27.0^{\circ} \mathrm{C}$, and March $26.4^{\circ} \mathrm{C}$ ) recorded in 2011 in NC. At days 3, 8, and 14 post-infection, saliva samples were collected as described in Dubrulle and colleagues (2009), and the titer was estimated by focus fluorescent assay on C6/36 Ae. albopictus cell culture. The transmission rate corresponds to the proportion of mosquitoes with infectious saliva (Dubrulle et al. 2009; Vazeille et al. 2010).

\section{Statistical analyses}

Descriptive statistics consisted of the calculation of median, minima, and maxima for continuous variables, and absolute numbers and proportions for categorical variables. Comparative analyses were performed on the basis of the Wilcoxon rank-sum test or the median test for continuous variables, and Fisher's exact test for categorical variables. A $p$ value $<0.05$ was considered statistically significant. Statistical analyses were performed using Stata software version 11.0 (Stata Corporation, College Station, TX).

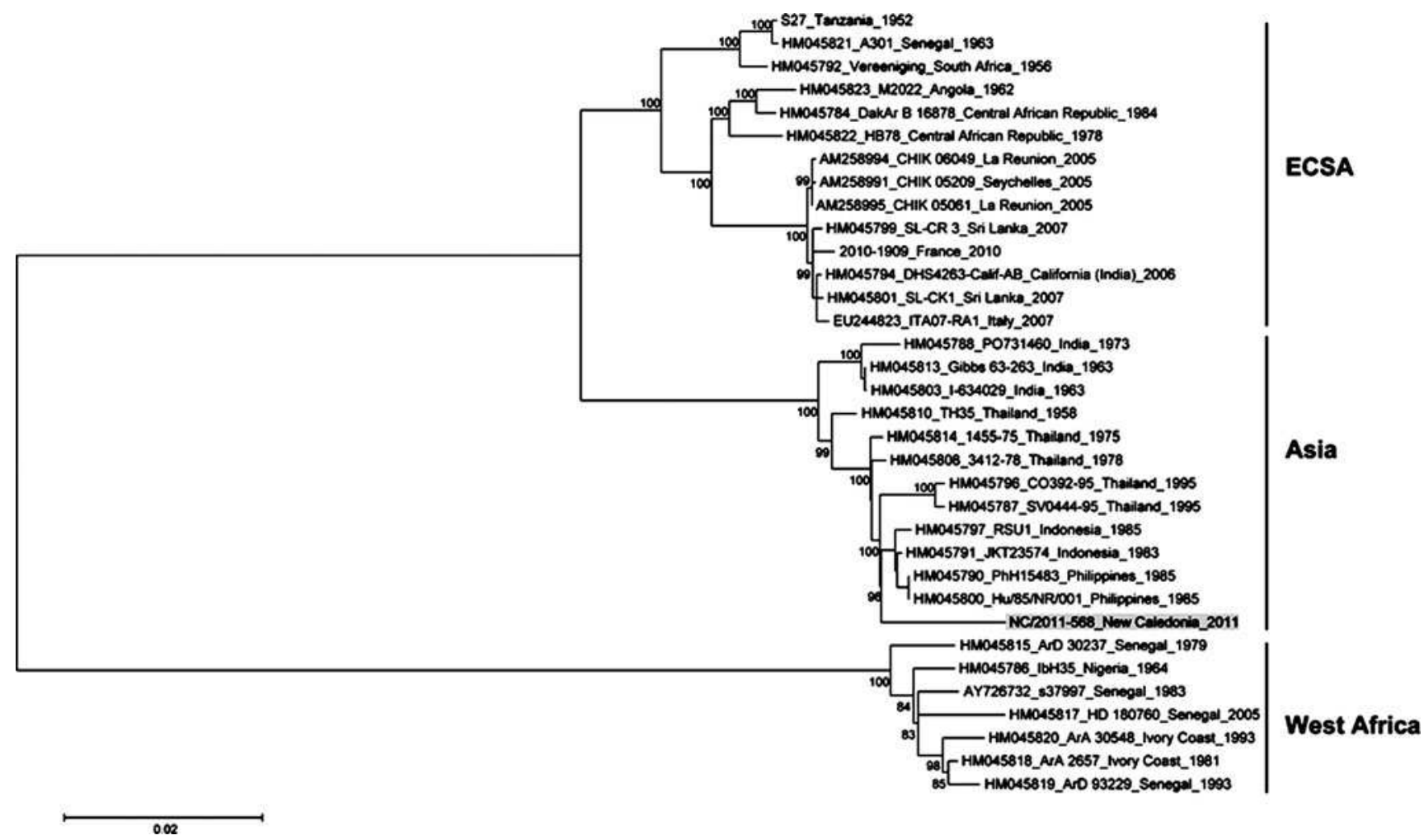

FIG. 1. Phylogenetic relationships among several CHIK viruses based on complete genome (11,238 nucleotides) analysis. Bootstrap support values (1000 replicates) are indicated at major nodes. The scale bar indicates the number of base substitutions per site (ECSA, East/Central/South Africa). 
Table 1. Characteristics of the Local Aedes aegypti Populations Tested

\begin{tabular}{lllc}
\hline Population & $\begin{array}{c}\text { Number of } \\
\text { generation }\end{array}$ & \multicolumn{1}{c}{ Localization } & $\begin{array}{c}\text { Susceptibility } \\
\text { to pyrethroids }\end{array}$ \\
\hline $163 / 11$ & F2 & Noumea surrounding & $92 \%$ \\
$174 / 11$ & F1 & Noumea & $85 \%$ \\
$282 / 10$ & & $\begin{array}{l}\text { New Caledonia } \\
\text { Ae. aegypti laboratory } \\
\end{array}$ & resistant strain \\
& & $58 \%$ \\
\hline
\end{tabular}

${ }^{a}$ Mortality observed after $60 \mathrm{~min}$ of exposure to papers impregnated with $0.05 \%$ deltamethrin.

\section{Results \\ Phylogenetic analysis of the New Caledonian CHIKV strain}

A molecular study of the CHIKV-NC strain (referenced as NC/2011-568) obtained from patient $C$ was conducted. Based on the complete genome nucleotide sequence, phylogenetic analysis demonstrated that the CHIKV-NC strain belonged to the Asian lineage, which was consistent with the return from Indonesia of patients A and B (Fig. 1). Furthermore, this isolate displayed $98.1 \%$ nucleotide identity with the other isolates of the Asian cluster. The amino acid sequence of $\mathrm{CHIKV-NC}$ revealed the presence of an alanine at position E1-226, an aspartic acid at position E2-60, and a threonine at positions E2-211 and E1-98. The E1-98T residue corroborates the phylogenetic analysis, as it has only been found in endemic Asian strains. It has been proposed that E1-98T may restrict the ability of Asian CHIKV strains to adapt to the vector Ae. albopictus (Tsetsarkin et al. 2011). The whole genome sequence of the CHIKV-NC strain was deposited in GenBank under accession no. HE806461.

\section{Mosquito investigation and susceptibility to pyrethroids}

The putative vector of CHIKV in NC is Ae. aegypti, since no other potential vector including Ae. albopictus has ever been described (Lee et al. 1982; Guillaumot 2005). Despite community awareness campaigns and intensive vector control carried out for many years in the context of dengue management, leading to a considerable decrease in vector density during the last decade, the species is still well established, particularly near human settlements. In 2011, relatively high Ae. aegypti densities were reported in urban and peri-urban areas of Noumea during the rainy season, from December to April (Guillaumot unpublished data).
Insecticide resistance tests showed that the two fieldcollected mosquito populations display a modified susceptibility to deltamethrin with an average mortality of $88.5 \%$ after $1 \mathrm{~h}$ of exposure (Table 1). By contrast, the laboratory strain is clearly resistant, with $58 \%$ mortality in the same conditions.

\section{CHIKV competence of local A. aegypti}

Vector competence refers to the biological ability of arthropods to acquire, maintain, and transmit pathogens. No significant differences were found either in the transmission rate, or in the number of viral particles in saliva, for the two CHIKV strains among the three populations (Tables 2 and 3). No relation could be established between these results and susceptibility to pyrethroids, although it has been stated that insecticide resistance may have an impact on several characteristics of vectors including vector competence (Rivero et al. 2010).

However, our results showed that local $A$. aegypti from NC could transmit both CHIKV strains efficiently (Fig. 2). At day 14 post-infection, the transmission rate of CHIKV-RE was higher than CHIKV-NC ( $p=0.002)$. Moreover, at days 3 and 14 post-infection, the number of CHIKV-RE viral particles delivered in saliva was higher compared to CHIKV-NC ( $p=0.0485$ and 0.019 , respectively). With the CHIKV-NC strain, no significant difference was found regarding the transmission rate and the number of viral particles secreted on days 3,8 , and 14 post-infection in A. aegypti ( $p=0.348$ and 0.979 , respectively). Corroborating previous studies of Aedes species (Dubrulle et al. 2009), we found that $A$. aegypti from NC was able to transmit both CHIKV strains as early as 3 days post-infection. Thus, the extrinsic incubation period (EIP) of both CHIKV strains in this vector can be considered as very short.

\section{Discussion}

We report here on the genotype of the CHIKV responsible for the first autochthonous cases in $\mathrm{NC}$, and show that local A. aegypti populations are highly competent for this virus.

Despite this high competence level, no large outbreak occurred in NC in a supposedly naive population (Alibert et al. 2011). Indeed, the short EIP obtained in the laboratory, and high mosquito densities (Guillaumot unpublished data), similar to those observed during the last dengue 4 episode in 2009 , were probably sufficient to sustain an outbreak. However, as shown by the vector competence results, the NC A. aegypti is more competent for CHIKV-RE from the ECSA genotype than CHIKV-NC from the Asian genotype, with higher transmission rates and viral loads in saliva. Even if the

Table 2. Median Value of Viral Particles in Saliva Collected from Aedes aegypti

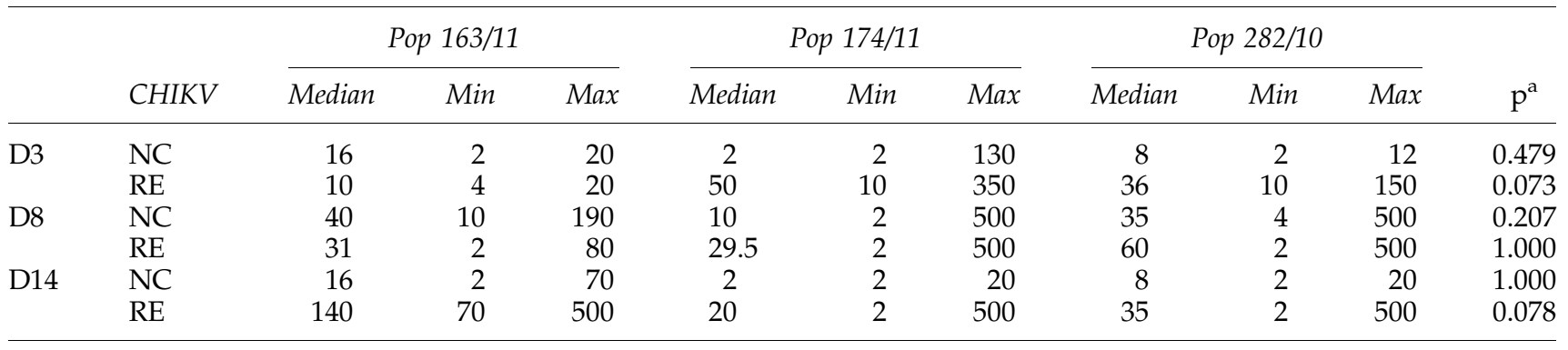

${ }^{a}$ Median test. Infectious titer: $10^{7} \mathrm{pfu} / \mathrm{mL}$.

CHIKV-NC corresponds to the strain NC 2011-568; CHIKV-RE corresponds to the strain 06.21 (harboring the mutation E1-A226V). 
Table 3. Transmission Rate of Chikungunya Virus in Aedes aegypti

\begin{tabular}{|c|c|c|c|c|c|c|c|c|c|c|c|}
\hline & \multirow[b]{2}{*}{ CHIKV } & \multicolumn{3}{|c|}{ Рор 163/11 } & \multicolumn{3}{|c|}{ Рор 174/11 } & \multicolumn{3}{|c|}{ Pop 282/10 } & \multirow[b]{2}{*}{$\mathrm{p}^{\mathrm{a}}$} \\
\hline & & $n$ inf. & n tot. & $\%$ & $n$ inf. & n tot. & $\%$ & $n$ inf. & $n$ tot. & $\%$ & \\
\hline \multirow[t]{2}{*}{ D3 } & $\mathrm{NC}$ & 8 & 15 & 53.3 & 7 & 14 & 50.0 & 6 & 15 & 40.0 & 0.806 \\
\hline & RE & 5 & 15 & 33.3 & 11 & 15 & 73.3 & 6 & 15 & 40.0 & 0.076 \\
\hline \multirow[t]{2}{*}{ D8 } & $\mathrm{NC}$ & 6 & 11 & 54.5 & 9 & 14 & 64.3 & 10 & 17 & 58.8 & 0.924 \\
\hline & $\mathrm{RE}$ & 8 & 14 & 57.1 & 6 & 13 & 46.2 & 8 & 14 & 57.1 & 0.855 \\
\hline \multirow[t]{2}{*}{ D14 } & $\mathrm{NC}$ & 4 & 6 & 66.7 & 3 & 15 & 20.0 & 3 & 6 & 50.0 & 0.144 \\
\hline & $\mathrm{RE}$ & 6 & 8 & 75.0 & 9 & 10 & 90.0 & 12 & 18 & 66.7 & 0.478 \\
\hline
\end{tabular}

${ }^{a}$ Fisher exact test. Infectious titer: $10^{7} \mathrm{pfu} / \mathrm{mL}$.

CHIKV-NC corresponds to the strain NC 2011-568; CHIKV-RE corresponds to the strain 06.21 (harboring the mutation E1-A226V).

$\mathrm{n}$ inf., number of infected females tested; $\mathrm{n}$ tot., total number of females tested.

CHIKV infectivity for $A$. aegypti was not influenced by the E2-I211T mutation, the E2-G60D mutation was an important determinant of CHIKV infectivity for $A$. aegypti (and A. albopictus) (Tsetsarkin et al. 2009). As the CHIKV-NC and CHIKV-RE strains used in this study were both harboring the E2-G60D mutation, we were unable to draw an accurate conclusion on the role of this mutation to explain differences in vector competence. Furthermore, at day 14 post-infection, the number of viral particles secreted by $A$. aegypti infected with the CHIKV-NC strain remained low. As the number of viral particles necessary to efficiently infect a human is unknown, this low number might be sufficient to contaminate and initiate an outbreak. However, saliva collected from mosquitoes infected with CHIKV-RE contains almost 10 times more viral particles, with probable consequences for the disease severity that was observed on Reunion Island (Gerardin et al. 2008). To feed on blood, A. aegypti injects saliva during the intradermal probing period, and is able to interrupt the intake of the blood meal when disturbed. Thus multiple feedings may lead to transmission of the virus to multiple hosts during the completion of one blood meal by a single mosquito (Platt et al. 1997). It is tempting to speculate that with a high viral load in saliva, A. aegypti infected with CHIKV-RE is able to deliver successive small quantities of infectious particles, while this scenario is less applicable to CHIKV-NC.

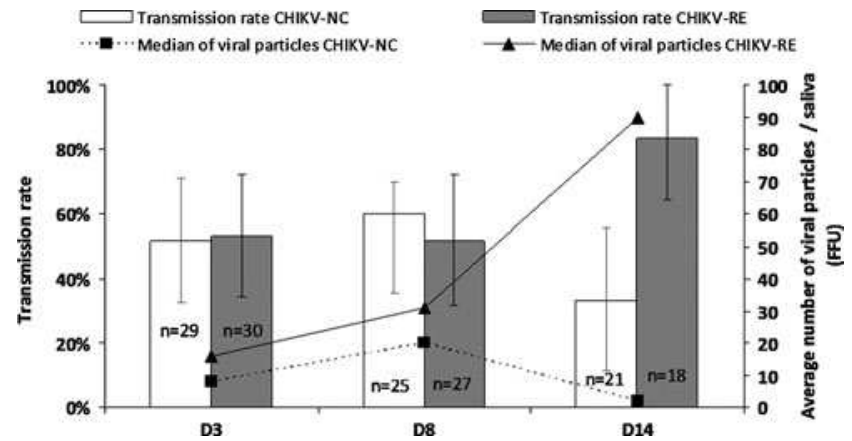

FIG. 2. Transmission rates and numbers of viral particles in the saliva of $A$. aegypti from NC after infection with the two CHIKV strains. Transmission potential was evaluated by estimating the number of infectious viral particles in excreted saliva by fluorescent foci units on C6/36 cells. The transmission rate is the ratio of the number of females positive for CHIKV in their saliva per the number of females tested ( $\mathrm{n}=$ number of females tested).
Vector control measures and the beginning of the cold season, combined with low transmission rates and low viral loads in saliva, might have been sufficient to limit CHIKV transmission. Other factors responsible for the emergence, including ecological factors, social parameters, vector/host associations, and host/virus genetic factors and their interactions, should be thoroughly investigated. This study also demonstrates that active foci of transmission or maintenance of Asian genotype strains can still persist and spread in the world via viremic travelers. Moreover, the potential to initiate an outbreak from virus vertically acquired from infected females should be also investigated. Whereas the ECSA genotype has widely spread in the world since 2006, Asian isolates still circulate and also represent a risk of emergence in regions where competent vectors exist, and where the human population is widely naive. As demonstrated during the recent emergence in Europe (Rezza et al. 2007; Grandadam et al. 2011), imported cases returning from endemic countries represent a risk for CHIKV emergence. Thus surveillance and diagnostic measures are essential to prevent this emergence.

\section{Conclusions}

The efficient CHIKV transmission that occurred in NC highlights its potential dissemination throughout the South Pacific region where it has never before been described, although A. aegypti is ubiquitous and might play the role of main vector. Even if the CHIKV transmission in NC ceased during the southern winter, CHIK cases could be reintroduced from Asia, and also from Reunion Island or Indian Ocean Islands (via air travel), where the CHIKV-RE strain is predominant. Thus the virus could propagate via viremic travelers to other Pacific islands, as previously observed for dengue epidemics (Li et al. 2010; Steel et al. 2010). Public health authorities in the Pacific community should pay attention to other mosquito species present in the South Pacific region that can act as efficient $\mathrm{CHIKV}$ vectors, such as A. albopictus and other species belonging to the scutellaris group (Guillaumot 2005). Physicians should also be aware to test for CHIK when patients present with dengue-like or influenza-like syndromes associated with arthralgias without respiratory symptoms.

\section{Acknowledgments}

We thank Sosiasi Kilama, Olivia O'Connor (Institut Pasteur of New Caledonia), Anubis Vega-Rua (Institut Pasteur, Paris), and the virology diagnostic laboratory staff from the Institut 
Pasteur of New Caledonia for technical assistance. We thank Scott C. Weaver for providing the CHIKV sequences of the Asian isolates used in this analysis. We are grateful to Suzanne Chanteau for scientific support and manuscript revision. We also thank Sylvia and Stephane Goiran and Anne Barthel for manuscript revision.

This work was supported by Institut Pasteur (New Caledonia and Paris), the Government of New Caledonia, and the French Institut de Veille Sanitaire (InVS, Saint Maurice, France).

\section{Author Disclosure Statement}

No competing financial interests exist.

\section{References}

Alibert A, Pfannstiel A, Grangeon JP. Chikungunya outbreak in New Caledonia in 2011, Status report as at 22 August 2011. Inform'Action 2011; 34:3-9.

de Lamballerie $X$, Leroy E, Charrel RN, et al. Chikungunya virus adapts to tiger mosquito via evolutionary convergence: a sign of things to come? Virol J 2008; 5:33-36.

Dubrulle M, Mousson L, Moutailler S, et al. Chikungunya virus and Aedes mosquitoes: saliva is infectious as soon as two days after oral infection. PLoS One 2009; 4:e5895.

Enserink M. Infectious diseases. Chikungunya: no longer a third world disease. Science 2007; 318:1860-1861.

Gerardin P, Barau G, Michault A, et al. Multidisciplinary prospective study of mother-to-child chikungunya virus infections on the island of La Réunion. PLoS Med 2008; 5:e60.

Grandadam M, Caro V, Plumet S, et al. Chikungunya virus, southeastern France. Emerg Infect Dis 2011; 17:910-913.

Guillaumot L. Arboviruses and their vectors in the Pacificstatus report. Pac Health Dialog 2005; 12:45-52.

Hall RA, Prow NA, Pyke AT. Ross River virus. In: Molecular Detection of Human Viral Pathogens. Liu D, ed. Boca Raton, FL: Taylor \& Francis, 2010:349-359.

Hapuarachchi HC, Bandara KB, Sumanadasa SD, et al. Reemergence of Chikungunya virus in South-east Asia: virological evidence from Sri Lanka and Singapore. J Gen Virol 2010; 91:1067-1076.

Lee DJ, Hicks MM, Griffiths M, et al. The Culicidae of the Australasian Region. Australian Government Publishing Service, Canberra, Australia. 1982:2.

Li DS, Liu W, Guigon A, et al. Rapid displacement of dengue virus type 1 by type 4, Pacific region, 2007-2009. Emerg Infect Dis 2010; 16:123-125.

Parola $\mathrm{P}$, de Lamballerie $\mathrm{X}$, Jourdan J, et al. Novel chikungunya virus variant in travelers returning from Indian Ocean islands. Emerg Infect Dis 2006; 12:1493-1499.

Pastorino B., Bessaud M, Grandadam M, et al. Development of a TaqMan RT-PCR assay without RNA extraction step for the detection and quantification of African Chikungunya viruses. J Virol Methods 2005; 124:65-71.

Pastorino B, Muyembe-Tamfum JJ, Bessaud M, et al. Epidemic resurgence of Chikungunya virus in democratic Republic of the Congo: identification of a new central African strain. J Med Virol 2004; 74:277-282.

Paupy C, Delatte H, Bagny L, et al. Aedes albopictus, an arbovirus vector: from the darkness to the light. Microbes Infect 2009; 11:1177-1185.
Platt KB, Linthicum KJ, Myint KS, et al. Impact of dengue virus infection on feeding behavior of Aedes aegypti. Am J Trop Med Hyg 1997; 57:119-125.

Rezza G, Nicoletti L, Angelini R, et al. Infection with chikungunya virus in Italy: an outbreak in a temperate region. Lancet 2007; 370:1840-1846.

Rivero A, Vezilier J, Weill M, et al. Insecticide control of vectorborne diseases: when is insecticide resistance a problem? PLoS Pathog 2010; 6:e1001000

Schuffenecker I, Iteman I, Michault A, et al. Genome microevolution of chikungunya viruses causing the Indian Ocean outbreak. PLoS Med 2006; 3:e263.

Schwartz O, Albert ML. Biology and pathogenesis of chikungunya virus. Nat Rev Microbiol 2010; 8:491-500.

Steel A, Gubler DJ, Bennett SN. Natural attenuation of dengue virus type-2 after a series of island outbreaks: a retrospective phylogenetic study of events in the South Pacific three decades ago. Virology 2010; 405:505-512.

Tsetsarkin KA, Chen R, Leal G, et al. Chikungunya virus emergence is constrained in Asia by lineage-specific adaptive landscapes. Proc Natl Acad Sci USA 2011; 108:78727877.

Tsetsarkin KA, McGee CE, Volk SM, et al. Epistatic roles of E2 glycoprotein mutations in adaptation of chikungunya virus to Aedes albopictus and Ae. aegypti mosquitoes. PLoS One 2009; 4:e6835.

Tsetsarkin KA, Vanlandingham DL, McGee CE, et al. A single mutation in chikungunya virus affects vector specificity and epidemic potential. PLoS Pathog 2007; 3:e201.

Vazeille-Falcoz M, Mousson L, Rodhain F, et al. Variation in oral susceptibility to dengue type 2 virus of populations of Aedes aegypti from the islands of Tahiti and Moorea, French Polynesia. Am J Trop Med Hyg 1999; 60:292-299.

Vazeille M, Mousson L, Martin E, et al. Orally co-infected Aedes albopictus from La Reunion Island, Indian Ocean, can deliver both dengue and chikungunya infectious viral particles in their saliva. PLoS Negl Trop Dis 2010; 4:e706.

Vazeille M, Moutailler S, Coudrier D, et al. Two Chikungunya isolates from the outbreak of La Reunion (Indian Ocean) exhibit different patterns of infection in the mosquito, Aedes albopictus. PLoS One 2007; 2:e1168.

Volk SM, Chen R, Tsetsarkin KA, et al. Genome-scale phylogenetic analyses of chikungunya virus reveal independent emergences of recent epidemics and various evolutionary rates. J Virol 2010; 84:6497-6504.

World Health Organization. Instructions for determining the susceptibility or resistance of adult mosquitoes to organochlorine, organophosphate and carbamate insecticides diagnostic test. WHO/VBC/81.806. Geneva; 1981.

Address correspondence to: Myrielle Dupont-Rouzeyrol Institut Pasteur de Nouvelle-Calédonie Laboratoire d'Epidémiologie Moléculaire 9-11 ave $P$. Doumer BP 61, 98845 Noumea New Caledonia

E-mail: mdupont@pasteur.nc 